\title{
A Note on Computing a Maximal Planar Subgraph using $P Q$-Trees
}

\author{
Michael Jünger, Sebastian Leipert, Petra Mutzel
}

\begin{abstract}
The problem of computing a maximal planar subgraph of a non planar graph has been deeply investigated over the last 20 years. Several attempts have been tried to solve the problem with the help of $P Q$-trees. The latest attempt has been reported by Jayakumar et al. [10].

In this paper we show that the algorithm presented by Jayakumar et al. is not correct. We show that it does not necessarily compute a maximal planar subgraph and we note that the same holds for a modified version of the algorithm presented by Kant [12]. Our conclusions most likely suggest not to use $P Q$-trees at all for this specific problem.

Keywords- $P Q$-Trees, Maximal Planar Subgraphs, Planarization.
\end{abstract}

\section{INTRODUCTION}

The minimum number of layers needed in the layout of printed circuit boards and integrated chips is equal to the thickness of the interconnection graph [15]. The thickness of a graph $G$ is the minimum number of planar subgraphs whose union is $G$. In VLSI design the thickness problem is approximated by successively subtracting large planar subgraphs from a given nonplanar graph. Another widely used method in VLSI design and Automatic Graph Drawing is to construct a planar subgraph from a given nonplanar graph by deleting a small number of edges and then to reinsert the removed edges, such that the number of edge crossings is small. However, the problem of finding the minimum number of edges that have to be removed from a given graph in order to obtain a planar subgraph, is known to be an $\mathcal{N} \mathcal{P}$-hard problem (see Garey and Johnson [7]).

Therefore, research has focused on computing maximal planar subgraphs. Let $G=(V, E)$ be a simple graph with $n$ vertices and $m$ edges then a planar subgraph $G^{\prime}$ of $G$ is a maximal planar subgraph, if for all edges $e \in G-$ $G^{\prime}$ the addition of $e$ to $G^{\prime}$ destroys planarity. Besides a trivial $\mathrm{O}(\mathrm{nm})$ algorithm that can be constructed using any $\mathrm{O}(n)$ planarity test, three different approaches are known for solving this problem.

Chiba, Nishioka and Shirakawa [3] presented an algorithm based on the path addition algorithm that computes a maximal planar subgraph in $\mathrm{O}(\mathrm{nm})$ time. Cai, Han, and Tarjan [2] presented later an $\mathrm{O}(m \log n)$ algorithm that is

Partially supported by DFG-Grant Ju204/7-2, Forschungsschwerpunkt "Effiziente Algorithmen für diskrete Probleme und ihre Anwendungen" and ESPRIT Long Term Research Project Nr. 20244 (ALCOM-IT).

M. Jünger is with the Institut für Informatik, Universität zu Köln, Pohligstr. 1, 50969 Köln, Germany. E-mail: mjuenger@informatik. uni-koeln.de

S. Leipert is with the Institut für Informatik, Universität zu Köln, Pohligstr. 1, 50969 Köln, Germany. E-mail: leipert@informatik.unikoeln.de

P. Mutzel is with the Max-Planck-Institut für Informatik, Im Stadtwald, 66123 Saarbrücken, Germany. E-mail: mutzel@mpi-sb.mpg.de based on the path addition algorithm as well. Di Battista and Tamassia [4] described an algorithm that checks in $\mathrm{O}(\log n)$ amortized time, whether an edge can be added to $G$ without destroying planarity, obtaining an $\mathrm{O}(m \log n)$ time algorithm as well.

Ozawa and Takahashi [16] have presented an $\mathrm{O}(\mathrm{nm})$ algorithm using the vertex addition algorithm. Jayakumar, Thulasiraman and Swamy [9] showed that in general this algorithm does not determine a maximal planar subgraph. Moreover, the resulting planar subgraph may not even contain all vertices. Jayakumar, Thulasiraman and Swamy [10] presented an algorithm called PLANARIZE that computes a spanning planar subgraph $G_{p}$ of $G$ in $\mathrm{O}\left(n^{2}\right)$ time. Furthermore, they present an algorithm called MAX-PLANARIZE that augments $G_{p}$ to a subgraph $G^{\prime}$ of $G$ by adding additional edges in $O\left(n^{2}\right)$ time. They claim that $G^{\prime}$ is a maximal planar subgraph of $G$ if $G_{p}$ (the result of phase 1 of the two phase algorithm) turns out to be biconnected. Kant [12] shows that this algorithm is incorrect, and suggests a modification of the second phase of the algorithm that augments $G_{p}$ to a maximal planar subgraph of $G$, even if $G_{p}$ is not biconnected, maintaining $O\left(n^{2}\right)$ time requirement.

In this article, we will point out a substantial flaw in both the original and the modified two phase algorithm that was not detected previously as well as new mistakes introduced by Kant. In section 2 we give a brief introduction on $P Q$-trees and the planarity test using this data structure. In section 3 the principle of the planarization algorithm using the $P Q$-trees is described. In section 4 we show that the algorithm of Jayakumar et al. [10] is incorrect giving a detailed description of the major mistake and discuss the attempt of Kant. In the last section we make some concluding remarks.

\section{Planarity test using $P Q$-trees}

We assume that the reader is familiar with the basic graph theoretic definitions as mentioned in [8]. A graph is planar, if it can be embedded in the plane without any edge crossings. A graph is obviously planar, if and only if its biconnected components are planar. We therefore assume that $G$ is biconnected. The planarity testing algorithm of Lempel, Even and Cederbaum [14] first labels the vertices of $G$ as $1,2 \ldots, n$ using an st-numbering (see [6]). A numbering of the vertices of $G$ by $1,2 \ldots, n$ is an st-numbering, if the vertices " 1 " and " $n$ " are adjacent and each other vertex $j$ is adjacent to two vertices $i$ and $k$ such that $i<j<k$. The vertex 1 is denoted by $s$ and the vertex $n$ is denoted by $t$. The $s t$-numbering induces an ori- 
entation of the graph, in which every edge is directed from the incident vertex with the higher $s t$-number towards the incident vertex with the lower $s t$-number. From now on we refer to the vertices of $G$ by their st-numbers and call an edge $(u, v)$, with $v<u$, incoming edge of $v$ and outgoing edge of $u$.

For $1 \leq k \leq n$, let $G_{k}$ denote the subgraph of $G$ induced by the vertex set $V_{k}:=\{1,2, \ldots, k\}$. The graph $G_{k}^{\prime}$ arises from $G_{k}$ as follows: For each edge $e=(u, v)$, where $v \in V_{k}$ and $u \in V \backslash V_{k}$, we introduce a virtual vertex $u_{e}$ with label $u$ and a virtual edge $\left(u_{e}, v\right)$. Let $B_{k}$ be a planar embedding of $G_{k}^{\prime}$ such that all virtual vertices are placed on the outer face. Then, $B_{k}$ is called a bush form. It has been shown by Lempel et al. [14] that $G$ is planar, if and only if for every $B_{k}, k=1,2, \ldots, n-1$, there exists a bush form $B_{k}^{\prime}$ isomorphic to $B_{k}$, such that all virtual vertices in $B_{k}^{\prime}$ labeled $k+1$ appear consecutively.

For an efficient computation of $B_{k}^{\prime}$, the $P Q$-tree technique [1] is applied. The $P Q$-tree $T_{k}$ corresponding to the bush form $B_{k}$ is a rooted ordered tree that consists of three types of nodes:

1. Leaves in $T_{k}$ represent virtual edges in $B_{k}$.

2. $P$-nodes in $T_{k}$ represent cutvertices in $B_{k}$.

3. $Q$-nodes represent maximal biconnected components in $B_{k}$.

The frontier of a $P Q$-tree is the sequence of all leaves of $T_{k}$ read from left to right. The frontier of a node $X$ is the sequence of its descendant leaves read from left to right.

Let $E_{k+1}$ denote the set of leaves in $T_{k}$ that correspond to the virtual vertices labeled $k+1$. A node $X$ is called full, if all leaves in its frontier are in $E_{k+1}$. A node $X$ is empty, if its frontier does not contain any leaf of $E_{k+1}$. Otherwise, $X$ is called partial. A node is called pertinent, if it is full or partial. The pertinent subtree is the smallest connected subtree that contains all leaves of $E_{k+1}$ in its frontier. The root of the pertinent subtree is called pertinent root. Two $P Q$-trees are equivalent, if one can be obtained from the other by one or more of the following operations:

1. Permuting the children of a $P$-node.

2. Reversing the order of the children of a $Q$-node.

These operations are called equivalence transformations and describe equivalence classes on the set of all $P Q$-trees. An equivalence class of $P Q$-trees corresponds to a class of permutations called the permissible permutations.

It has been shown by Booth and Lueker [1] that $B_{k}^{\prime}$ exists if and only if $T_{k}$ can be converted into an equivalent $P Q$ tree $T_{k}^{\prime}$ such that all pertinent leaves appear consecutively in the frontier of $T_{k}^{\prime}$. Booth and Lueker [1] have defined a set of patterns and replacements called templates that can be used to reduce the $P Q$-tree such that the leaves corresponding to edges of the set $E_{k+1}$ appear consecutively in all permissible permutations. To construct $T_{k+1}$ from $T_{k}$ they first reduce $T_{k}$ by use of the templates and then replace all leaves corresponding to virtual edges incident to virtual vertices labeled $k+1$ by a $P$-node, whose children are the leaves corresponding to the incoming edges of the vertex $k+1$ in $G$.

The planarity testing algorithm now starts with $T_{1}$ and constructs a sequence of $P Q$-trees $T_{1}, T_{2}, \ldots$ If the graph is planar, the algorithm terminates after constructing $T_{n-1}$. Otherwise it terminates after detecting the impossibility of reducing some $T_{k}, 1 \leq k<n$.

\section{PRINCIPle OF AN APPROACH FOR PLANARIZATION}

The basic idea of a planarization algorithm using $P Q$ trees presented by Jayakumar et al. [10] is to construct the sequence of $P Q$-trees $T_{1}, T_{2}, \ldots, T_{n-1}$ by deleting an appropriate number of pertinent leaves every time the reduction fails such that the resulting $P Q$-tree becomes reducible. In every step of the algorithm PLANARIZE, a maximal consecutive sequence of pertinent leaves is computed by using a $[w, h, a]$-numbering (see [10]). All pertinent leaves that are not adjacent to the maximal pertinent sequence are removed from the $P Q$-tree in order to make it reducible. Hence the edges corresponding to the leaves are removed from $G$ and the resulting graph $G_{p}$ is planar.

It has been shown by Jayakumar et al. [10] that the graph $G_{p}$ computed by PLANARIZE is not necessarily maximal planar. The authors therefore suggest to apply a second phase called MAX-PLANARIZE, also based on $P Q$-trees. Knowing which edges have been removed from $G$ to construct $G_{p}$, edges from $G-G_{p}$ are added back to $G_{p}$ in the second phase without destroying planarity.

During the reduction of a vertex $v$, there may exist nonpertinent leaves that are between a pertinent leaf $l_{v}$ and its maximal pertinent sequence in all permissible permutations of the $P Q$-tree $T_{v-1}$. This maximal pertinent sequence has been determined with the help of the $[w, h, a]$-numbering. In order to make the tree $T_{v-1}$ reducible, the leaf $l_{v}$ is removed from the tree and the corresponding edge is removed from the graph $G$, guaranteeing that the subgraph $G_{p}$ will be planar. However, it may occur that the nonpertinent leaves that are positioned between $l_{v}$ and its maximal pertinent sequence in $T_{v-1}$, are removed as well from a tree $T_{k}, v \leq k<n$, in order to obtain reducibility. Therefore, there is no need to remove the edge corresponding to $l_{v}$ from the graph $G$.

In order to find leaves such as $l_{v}$, Jayakumar et al. [10] use the algorithm MAX-PLANARIZE. In step $i$, both PLANARIZE as well as MAX-PLANARIZE reduce the same vertex $i$. The difference between the $P Q$-trees in the two algorithms is, according to the authors, that all leaves that have been deleted in PLANARIZE are ignored in MAX-PLANARIZE from the moment they are introduced into the tree until they get pertinent. This causes the nonpertinent leaves between the pertinent leaf $l_{v}$ and its maximal pertinent sequence to be ignored. Hence $l_{v}$ is adjacent to its maximal pertinent sequence and the corresponding edge can be added back to $G_{p}$, while the leaves between $l_{v}$ and the maximal pertinent sequence are removed from the $P Q$-tree.

\section{ON THE INCORRECTNESS OF THE ALGORITHM}

While some incorrect facts of the approach of Jayakumar et al. have been described in a technical report by Kant [12], 
who attempted to correct the algorithm, a major problem has not been detected.

Jayakumar et al. assume that the maximal planar subgraph $G_{p}$ is biconnected for the correct application of the Lempel-Even-Cederbaum algorithm. Furthermore, as they have stated correctly, this is necessary in order to have an st-numbering. Nevertheless, the $P Q$-trees in MAX-PLANARIZE are constructed according to the stnumbering that was computed for the graph $G$.

As a matter of fact, the $s t$-numbering of $G$ does not imply an st-numbering of any subgraph $G_{p}$ even if the subgraph $G_{p}$ is biconnected. This results in two problems, of which one is crucial and cannot be dealt with even by the ideas described by Kant [12].

Both problems are based on the fact that during the application of PLANARIZE for some vertices of $V$ all incoming edges may be deleted from the graph while the resulting graph $G_{p}$ stays biconnected.

Let $v \in V$ be such a node with no incoming edges in $G_{p}$. Since $G_{p}$ is biconnected, $v$ must have at least two outgoing edges $\left(v, u_{1}\right)$ and $\left(v, u_{2}\right)$. Let $w \in V$ be a vertex in $G$ such that $u_{1}, u_{2}<w<v$. Thus the leaves corresponding to the outgoing edges of $w$ are reduced before the leaves of $v$. Let $T_{w-1}$ be the $P Q$-tree during the application of MAXPLANARIZE, in which the relevant leaves corresponding to the outgoing edges of $w$ have to be reduced. Assume that the leaves of both nodes $w$ and $v$ are on the outer face of the same biconnected component of the bush form that corresponds to the $P Q$-tree $T_{w-1}$. Assume further that one designated leaf $w_{k+1}$ of the vertex $w$ is separated by the leaves $v_{1}$ and $v_{2}$ corresponding to $\left(v, u_{1}\right)$ and $\left(v, u_{2}\right)$ from the leaves $w_{1}, w_{2}, \ldots, w_{k}$, where the latter form the maximal pertinent sequence (see Figure 1 for an illustration).

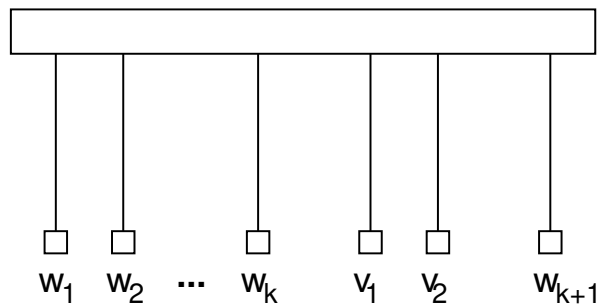

Fig. 1. Leaf $w_{k+1}$ is separated by $v_{1}$ and $v_{2}$ from its maximal pertinent sequence $w_{1}, w_{2}, \ldots, w_{k}$.

If $\left(v, u_{1}\right)$ and $\left(v, u_{2}\right)$ are the only outgoing edges of $v$ in $G_{p}$, then the leaves $v_{1}$ and $v_{2}$ will be changed during the reduction of the $P Q$-tree $T_{v-1}$ into a $P$-node with leaves corresponding to edges in $E \backslash E_{p}$. If none of the incoming edges of $v$ is added to $G_{p}$ in a $P Q$-tree $T_{i}, v<i<n$, the edge corresponding to the leaf $w_{k+1}$ can be added to the graph $G_{p}$ without destroying planarity. Hence, the resulting graph $G_{p}$ is not a maximal planar subgraph.

We now consider the second problem. The planarization algorithm of Jayakumar et al. [10] does not obey an important invariant implied by the following lemma, shown by Even [5].

Lemma IV.1: Let $G=(V, E)$ be a planar graph with an $s t$-numbering and let $1 \leq k \leq n$. If the edge $(t, s)$ is drawn on the boundary of the outer face in an embedding of $G$, then all vertices and edges of $G-G_{k}$ are drawn in the outer face of the plane subgraph $G_{k}$ of $G$.

This result allowed Lempel et al. to transform the problem of planarity testing to the construction of a sequence of bush forms $B_{k}, 1 \leq k \leq n$. For a planar graph $G$, edges and vertices that have not been introduced into the current subgraph $G_{k}$ are always embedded into the outer face of $G_{k}$.

The approach of Jayakumar et al. [10] does not obey this invariant in the second phase. There exist edges that have to be embedded into an inner face of some $G_{k}$, even if $(t, s)$ is drawn on the outer face. Due to the above lemma, the correction step MAX-PLANARIZE only considers edges for reintroduction into the planar subgraph $G_{p}$ that are on the outer face of the current graph $G_{k}$. Since the numbering that is used to determine the order in which the vertices are reduced does not correspond to an st-numbering of $G_{p}$ in general, the algorithm of Jayakumar et al. [10] ignores edges that can be added into an inner face of the embedding of a current graph $G_{k}$ without destroying planarity. This fact is fatal, as we are about to show now.

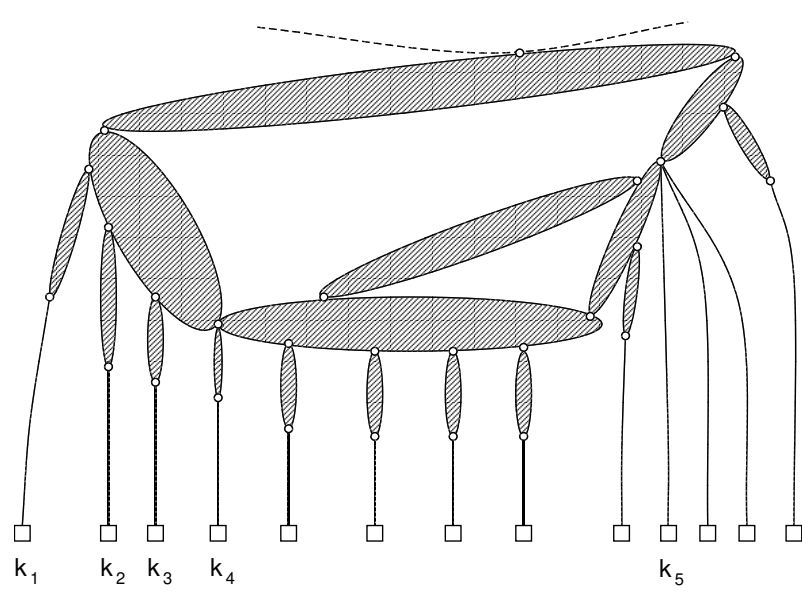

Fig. 2. Part of a bush form $B_{k-1}$

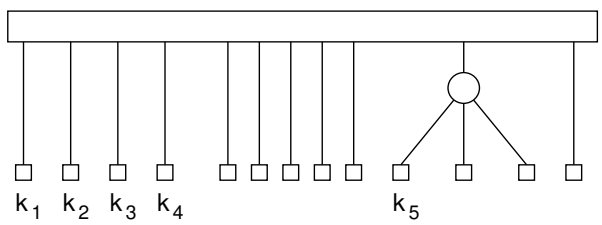

Fig. 3. Part of a $P Q$-tree corresponding to bush form $B_{k-1}$

In Figure 2, a part of a bush form $B_{k-1}, 1<k \leq n$, of a graph $G$ is shown. The virtual vertices corresponding to the vertex $k$ are labeled $k_{1}, k_{2}, \ldots, k_{5}$ and all other virtual vertices are left unlabeled. The corresponding part of the $P Q$-tree is shown in Figure 3. Obviously, there do not exist any reversions or permutations such that the virtual vertices of $k$ occupy consecutive positions. Hence, the graph $G$ is not planar. Applying the $[w, h, a]$-numbering of Jayakumar et al. [10] allows us to delete the virtual vertex 


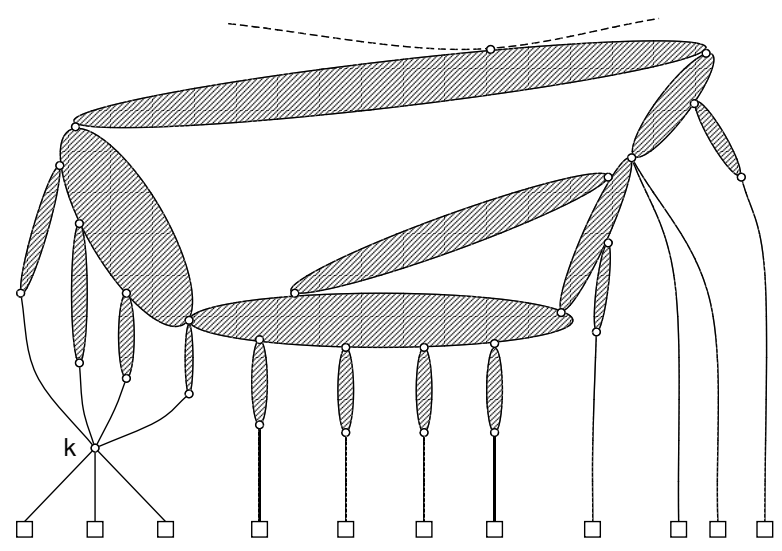

Fig. 4. Part of a bush form $B_{k}$

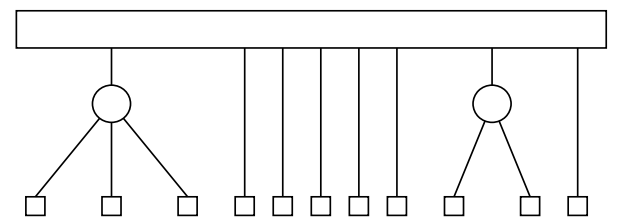

Fig. 5. Part of a $P Q$-tree corresponding to bush form $B_{k}$

$k_{5}$ and to reduce the other four vertices $k_{1}, k_{2}, k_{3}, k_{4}$. The resulting bush form $B_{k}$ is planar and the relevant part is shown in Figure 4. Figure 5 shows the corresponding part of the $P Q$-tree. Assume now that all descendants of $k$ have to be removed from the $P Q$-tree in a later step. Hence all incoming edges incident on $k$ are removed from the tree. Now assume further that there exists a path $v_{1}, v_{2}, \ldots, v_{l}$ in $G_{p}$ such that

- for all $i, j, 1 \leq i<j \leq l$ the inequality $v_{i}<v_{j}$ holds,

- the edge $\left(v_{2}, v_{1}\right)$ corresponds to one of the leaves that are between the leaf $k_{5}$ and the maximal pertinent sequence $k_{1}, k_{2}, k_{3}, k_{4}$ in all $P Q$-trees equivalent to $T_{k-1}$,

- $v_{l}=t$.

This path guarantees that all outgoing edges of the vertex $k$ cannot be embedded into the outer face of the embedding of $B_{k-1}$ without crossing an edge on this path. Hence the edge $e_{k_{5}}$ corresponding to the leaf $k_{5}$ is not considered by the algorithm MAX-PLANARIZE as being an edge that does not destroy planarity. Therefore, $e_{k_{5}}$ is not added back to the planar subgraph $G_{p}$.

Nevertheless adding the edge $e_{k_{5}}$ to $G_{p}$ may not destroy planarity of $G_{p}$ as is shown in our example in Figure 6. Since all incoming edges of the vertex $k$ have been deleted by PLANARIZE and are not added back by MAXPLANARIZE, it may be possible to swap the vertex $k$ into an inner face of the embedding of $B_{k}$ such that the virtual vertex $k_{5}$ can be identified with $k$ and the edge $e_{k_{5}}$ is embedded into the bush form $B_{k}$ without destroying planarity.

Therefore, the strategy of using $P Q$-trees presented by Jayakumar et al. [10] does not compute a maximal planar subgraph in general. Furthermore, we point out that the same problem holds for the modified version of this algorithm, presented by Kant [12]. This version follows a

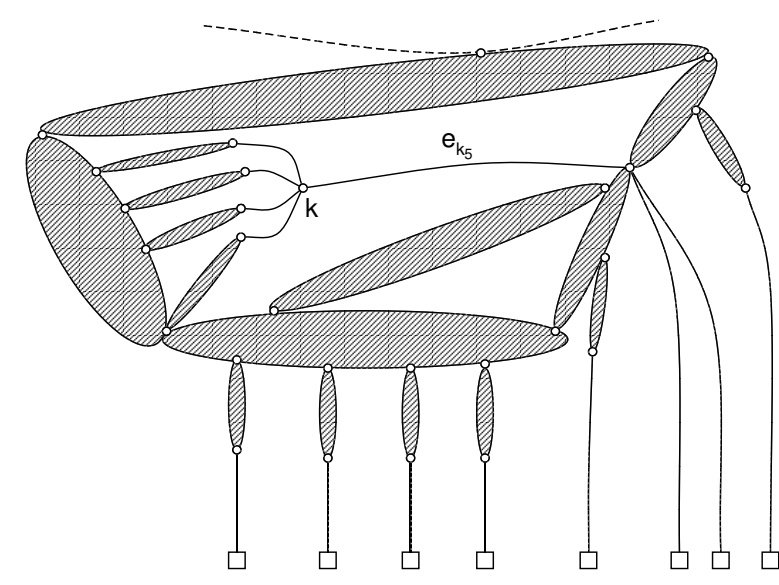

Fig. 6. Part of a bush form $B_{k}$ with $e_{k_{5}}$ embedded

similar strategy of computing a spanning planar subgraph $G_{p}$ using PLANARIZE and then adding edges that do not destroy planarity in a second phase. The order of reductions that is used to insert vertices into existing bush forms is the same as the one implied by the st-numbering on $G$. Hence this approach is not able to compute a maximal planar subgraph for the same reason.

Summarizing, we state the following lemma.

Lemma IV.2: Let $G=(V, E)$ be a nonplanar graph. Let $G_{p}=\left(V, E_{p}\right), E_{p} \subseteq E$, be a planar subgraph of $G$, such that $G_{p}$ was obtained from $G$ by

1. computing an st-numbering for all vertices and

2. applying the algorithm of Lempel et al. [14] constructing a sequence of bush forms $B_{k}, 1 \leq k \leq n$, by embedding a maximal number of outgoing edges of a vertex $k, 1<k \leq n$, in the outer face of $B_{k-1}$ without crossings, deleting all other outgoing edges of $k$.

Let $G_{p}^{\prime}=\left(V, E_{p}^{\prime}\right)$, be a planar subgraph of $G$ such that

1. $E_{p} \subseteq E_{p}^{\prime} \subseteq E$,

2. the graph $G_{p}^{\prime}$ is computed by constructing a sequence of bush forms $B_{k}^{\prime}, 1 \leq k \leq n$, based on the st-numbering used for determining $G_{p}$, and possibly embedding outgoing edges $e \in E \backslash E_{p}$ of every vertex $k, 1<k \leq n$, without crossings in the outer face of $B_{k-1}$.

Then the subgraph $G_{p}^{\prime}$ is not necessarily maximal planar.

Proof: Clear from the discussion above.

Considering a computation of an st-numbering for the planar subgraph $G_{p}$ in order to augment $G_{p}$ to a maximal planar subgraph of $G$ and then constructing a sequence of bush forms $B_{k}^{\prime}, 1 \leq k \leq n$, is aggravated by the fact that the graph $G_{p}$ is not biconnected in general. Furthermore, the difference between the bush forms of the first phase and the second phase may result in the deletion of the edges of $G_{p}$ as soon as edges of $E \backslash E_{p}$ are added to $G_{p}$. Adding an edge $e \in E \backslash E_{p}$ to $G_{p}$ is able to change the corresponding bush form in such a way, that the pertinent leaves corresponding to the outgoing edges of some node $v$ in $E_{p}$ cannot form a consecutive sequence in any permissible permutations.

If the $s t$-numbering of $G$ is as well an $s t$-numbering of $G_{p}$, the counterexamples given by Kant [12] show that MAX- 
PLANARIZE is not even correct for this special case. However, as has been shown in [13], [11], the modifications suggested by Kant [12] do not correct the algorithm presented by Jayakumar et al. [10].

Kant [12] suggested a correction of the second phase by introducing sequence indicators and by delaying the decision, whether a deleted leaf can be added back to $G_{p}$, until enough information is available. As has been laid out in [13], [11], this approach does not succeed for three main reasons.

1. If there are several deleted leaves corresponding to the outgoing edges of some vertex $v \in V$, the approach considers more edges for reintroduction than can actually be added without destroying planarity.

2. If edges are added back into the graph $G_{p}$, the set of permissible permutations of the corresponding $P Q$-tree is not restricted in a proper way.

3. If there are several deleted leaves corresponding to the outgoing edges of different vertices that can be added back to $G_{p}$ without destroying planarity, the approach does not consider all edges for reintroduction. The algorithm therefore does not necessarily compute a maximal planar subgraph.

The first two problems have been shown to be solvable by Leipert [13], but the last still remains unsolved.

\section{Concluding Remarks}

In this paper we showed that the attempt of Jayakumar et al. [10] to solve the maximal planar subgraph problem with $P Q$-trees is not correct. The problem is due to the fact that an important invariant for planarity testing is ignored. We have further noted that even a corrected version of the algorithm applied in the best possible case, where the stnumbering of a graph $G$ is as well an st-numbering of the planar subgraph $G_{p}$, is not correct.

Since this best case is a very rare case and since the modifications for the solved problems (see [13]) are far beyond any reasonable implementation, we doubt that a useful algorithm based on the strategy presented by Jayakumar et al. [10] can be found.

\section{REFERENCES}

[1] K.S. Booth and G.S. Lueker. Testing for the consecutive ones property, interval graphs, and graph planarity using PQ-tree algorithms. Journal of Computer and System Sciences, 13:335$379,1976$.

[2] J. Cai, X. Han, and R. E. Tarjan. An $O(m \log n)$-time algorithm for the maximal planar subgraph problem. SIAM Journal of Comput., 22:1142-1162, 1993.

[3] T. Chiba, I. Nishioka, and I. Shirakawa. An algorithm for maximal planarization of graphs. In Proceedings on the 1979 IEEE International Symposium on Circuits and Systems, pages 336441, 1979.

[4] G. Di Battista and R. Tamassia. Incremental planarity testing. In Proceedings on the 30th Annual IEEE Symposium on Foundations of Computer Science, North Carolina, pages 436-441, 1989 .

[5] S. Even. Graph Algorithms. Computer Science Press, Potomac, Maryland, 1979.

[6] S. Even and R. E. Tarjan. Computing an st-numbering. Theoretical Computer Science, 2:339-344, 1976.

[7] M. R. Garey and D. S. Johnson. Computers and Intractability: A Guide to the Theory of NP-completeness. Freeman \& Co., San Francisco, 1979.
[8] F. Harary. Graph Theory. Addison Wesley, 1969.

[9] R. Jayakumar, K. Thulasiraman, and M.N.S. Swamy. On maximal planarization of non-planar graphs. IEEE Transactions on Circuits Systems, 33(8):843-844, 1986.

[10] R. Jayakumar, K. Thulasiraman, and M.N.S. Swamy. On $O\left(n^{2}\right)$ algorithms for graph planarization. IEEE Transactions on Computer-Aided Design, 8(3):257-267, March 1989.

[11] M. Jünger, S. Leipert, and P. Mutzel. On computing a maximal planar subgraph using PQ-trees. Technical Report 96.227, Institut für Informatik, Universität zu Köln, 1996.

[12] G. Kant. An $O\left(n^{2}\right)$ maximal planarization algorithm based on PQ-trees. Technical Report RUU-CS-92-03, Department of Computer Science, Utrecht University, 1992.

[13] S. Leipert. Berechnung maximal planarer Untergraphen mit Hilfe von PQ-Bäumen. Master's thesis, Institut für Informatik der Universität zu Köln, 1995.

[14] A. Lempel, S. Even, and I. Cederbaum. An algorithm for planarity testing of graphs. In Theory of Graphs: International Symposium: Rome, July 1966, pages 215-232. Gordon and Breach, New York, 1967.

[15] T. Lengauer. Combinatorial Algorithms for Integrated Circuit Layout. Wiley, 1990.

[16] T. Ozawa and H. Takahashi. A graph-planarization algorithm and its application to random graphs. In Graph Theory and Algorithms, volume 108 of Lecture Notes in Computer Science, pages 95-107, 1981. 